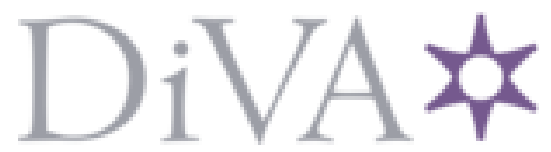

http://www.diva-portal.org

\title{
Postprint
}

This is the accepted version of a paper presented at Electrical Power and Energy Conference (EPEC 2017), Saskatoon, Saskatchewan, Canada October 22-25, 2017.

Citation for the original published paper:

Mahseredjian, J., Haddadi, A., Hooshyar, H., Vanfretti, L., Dufour, C. (2017)

An active distribution network model for smart grid control and protection studies: model validation progress.

In: IEEE

N.B. When citing this work, cite the original published paper.

(C) 2017 IEEE. Personal use of this material is permitted. Permission from IEEE must be obtained for all other uses, in any current or future media, including reprinting/republishing this material for advertising or promotional purposes, creating new collective works, for resale or redistribution to servers or lists, or reuse of any copyrighted component of this work in other works.

Permanent link to this version:

http://urn.kb.se/resolve?urn=urn:nbn:se:kth:diva-213006 


\section{An Active Distribution Network Model for Smart Grid Control and Protection Studies-Model Validation Progress}

\author{
Aboutaleb Haddadi \\ Jean Mahseredjian \\ Montréal Polytechnique \\ Montréal, QC, Canada \\ aboutaleb.haddadi@polymtl.ca \\ jean.mahseredjian@polymtl.ca
}

\author{
Hossein Hooshyar \\ KTH \\ Stockholm, Sweden \\ hosseinh@,kth.se
}

\author{
Luigi Vanfretti \\ Rensselaer Polytechnic Institute \\ Troy, NY, USA \\ luigi.vanfretti@gmail.com
}

\author{
Christian Dufour \\ Opal-RT Technologies \\ Montréal, QC, Canada \\ christian.dufour@,opal-rt.com
}

\begin{abstract}
This paper presents the implementation of an Active Distribution Network (ADN) model and its qualitative assessment using different off-line and real-time simulation tools. The objective is to provide software-to-software verification for the establishment of the model as a potential benchmark. Expanding upon the authors' previous work [7], this paper provides additional simulation results, cross-examination of the models, and presents the latest modifications incorporated to address practical considerations. The ADN has multiple voltage levels and features various types of distributed energy resources including solar, wind generators, and storage. It further incorporates control and protection schemes for distributed energy resource units and loads; hence, it adequately represents the complex dynamics of an active distribution grid compared to the existing distribution grid test cases in the literature. As such, the test system can be used by other researchers to test numerical methods and conduct research on Smart Grid control, protection, and dynamic studies.
\end{abstract}

Keywords: Active distribution network model, power system simulation, power system modeling, active distribution network, smart grid, control, protection.

\section{INTRODUCTION}

$\mathrm{P}$ OWER system test cases for simulation studies of active distribution grids are rare. Distribution grid benchmarks in the literature [1]-[4] cannot adequately represent the complex dynamics of an active distribution grid because they often operate at a single voltage level and lack components such as Distributed Energy Resources (DER) and protection systems. Reference [1] has presented a medium voltage (MV) and a low voltage (LV) distribution network benchmark for DER integration; both benchmarks operate at a single voltage level and do not contain DER units. Reference [2] has provided five distribution test feeders; although some of them feature two voltage levels, they lack DER units and, hence, do not represent an active grid. Details of these test feeders are available online at [3]. Reference [4] has presented a test distribution feeder which operates at a single voltage level and lacks DER units.

To address the abovementioned gap in the literature, [5]-[8]

Paper submitted to the Electrical Power and Energy Conference (EPEC2017), Saskatoon, SK, Canada, October 22-25, 2017. have presented an Active Distribution Network (ADN) model which features various voltage levels and DER unit models including control and protection schemes. Reference [5] has presented a MATLAB/Simulink implementation of the model that has been modified for use in the Opal-RT real-time simulator using the stub-line simulation technique. Reference [6] has shown that the stub-line implementation suffers from accuracy issues and presented another implementation using the state-space nodal analysis method (SSN) [9] to address the accuracy problem. Reference [6] has further developed an offline (non-real-time) implementation of the ADN that uses standard Simulink solvers and Simscape Power Systems (SPS) models [10]. The paper has shown that the results of the SSN and SPS implementations match closely. To further verify the ADN model implementations, [7] has presented another implementation within the EMTP-RV software environment for off-line simulations and compared the results to the SSN implementation. The paper has shown that the results from the EMTP and SSN implementations are adequate, but present inconsistencies due to modeling differences. The abovementioned model implementations are available online in the Github platform [8].

This paper presents the latest version of the ADN which improves the consistency of simulation results and features additional protection functions. To achieve a higher consistency, the models have been decomposed into components, and a component-by-component crossexamination has been performed between the EMTP-RV and SSN implementations. Next, the entire model has been crossexamined. This paper also presents the three-phase power flow solution of the ADN.

The model presented in this paper and its simulation with different tools provides the software-to-software verification basis for the establishment of the model as a potential benchmark. The model can be used by other researchers for testing numerical methods and conducting research on Smart Grid, control, protection, and dynamic studies.

\section{The ACtive Distribution Network MODEL}

Fig. 1 shows a single line diagram of the ADN which comprises 79 unbalanced buses. The model includes a $220-\mathrm{kV}$ transmission grid (HV), a $36-\mathrm{kV}$ medium-voltage (MV), a 6.6- 


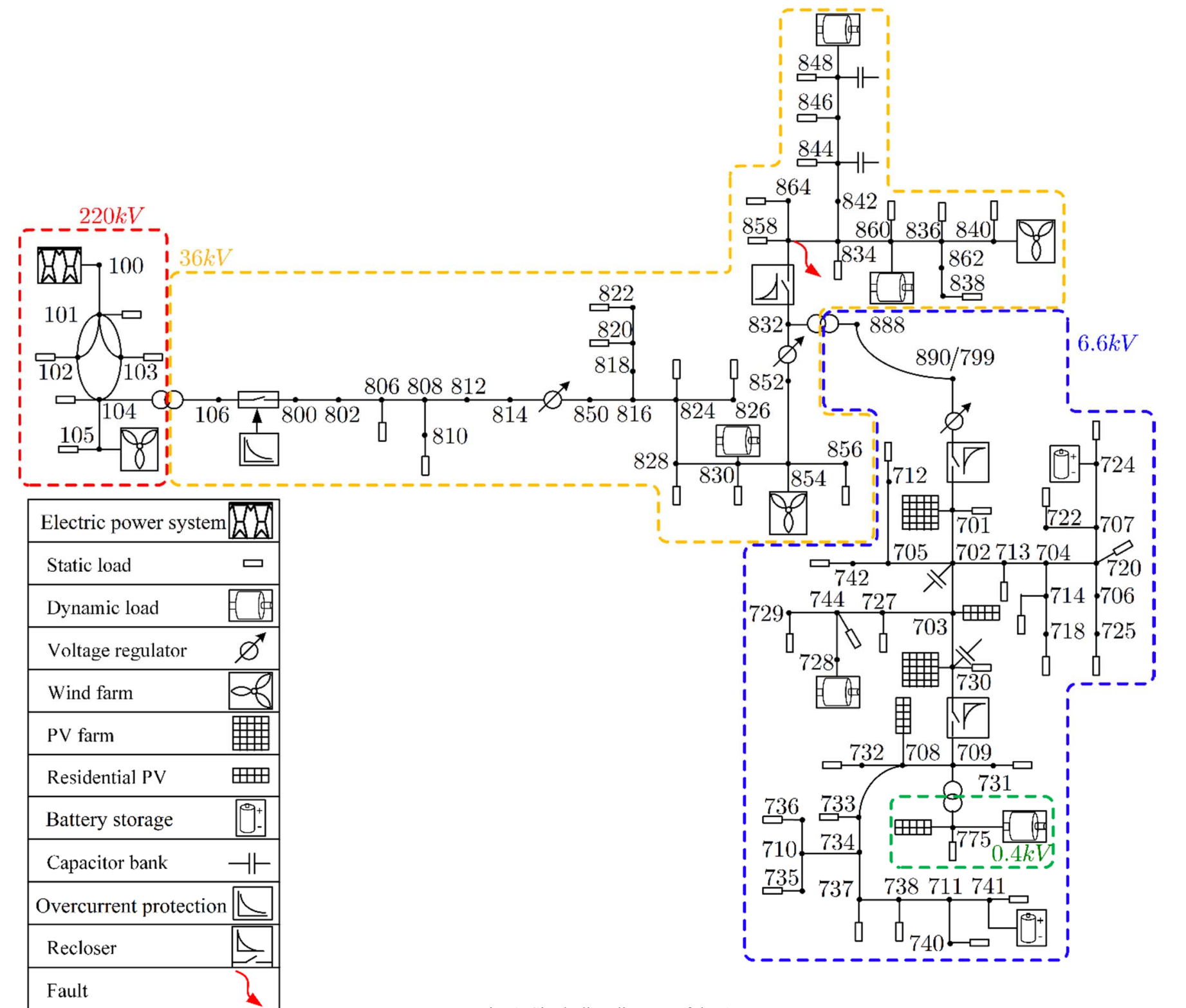

Fig. 1. Single-line diagram of the ADN.

$\mathrm{kV}$ low-voltage (LV), and a $0.4-\mathrm{kV}$ residential LV grid. The total generation is $200 \mathrm{MW}$, and the overall load of the modelled system is $191 \mathrm{MW}$. The generation and load of the ADN (MV, LV, and residential LV grids) represent a total DER generation of $5 \mathrm{MW}$ and load of $4.1 \mathrm{MW}+\mathrm{j} 1.9 \mathrm{MVar}$; hence, the ADN can be operated as a micro grid. The DER includes wind, solar, and storage component models. The model of the DERs includes power electronic interfaces, control, and protection schemes. System loads include both three-phase and single-phase static loads as well as motor loads. The ADN model also includes voltage, current, and frequency protection schemes for DER units, feeders, and loads. Reference [5] has presented the details of the component models. It should be mentioned that the ADN model presented in this paper uses accurate time-domain models for all components and, therefore, may be used for the simulation of fast electromagnetic transient events under adequate assumptions ${ }^{1}$.

\footnotetext{
${ }^{1}$ Note that certain components and portions of the model are represented with
}

Reference [6] has developed an implementation of the test ADN for real-time simulations in the Opal-RT simulator employing the SSN technique. Reference [7] has developed an implementation within EMTP-RV for off-line simulations; it contains no artificial delay blocks and uses an iterative solver for nonlinear functions. The paper has shown that the simulation results of the SSN and EMTP-RV are adequate, but present inconsistencies.

This paper presents the latest implementation of the ADN in EMTP-RV and using SSN. The latest version incorporates additional protection functions and achieves a better match between the results of the two implementations. The next section presents these modifications.

\section{MODIFICATIONS}

The latest version of the ADN incorporates three main modifications with respect to the older version presented in [6]

lumped parameter models, and thus, some electromagnetic transient phenomena cannot be represented without modifying the model accordingly. 
and [7] as follows.

\section{A. Overcurrent Protection for Motor Loads}

A time-definite overcurrent protection has been added to motor loads (single- and three-phase) which disconnects the load if the terminal current exceeds $5 \mathrm{pu}$ for more than $1 \mathrm{~s}$. The reconnection time of motor loads have also been changed to $25 \mathrm{~s}$. In three-phase motor loads, the overcurrent protection measures the positive sequence current whereas in singlephase motors, it measures the phase current.

(a)

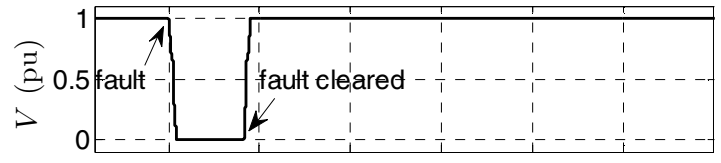

(b)

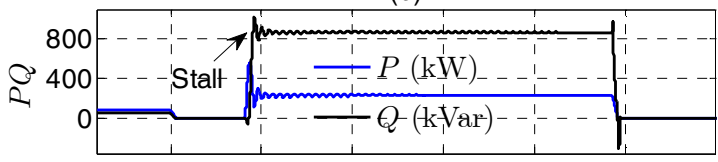

(c)

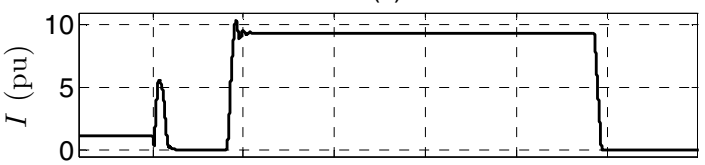

(d)

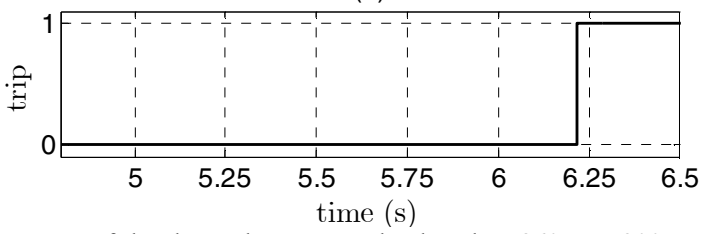

Fig. 2. Response of the three-phase motor load on bus 860 to a $200 \mathrm{~ms}$ threephase fault at its terminals: (a) terminal voltage of the motor in pu, (b) real and reactive power consumption in $\mathrm{kW}$ and $\mathrm{kVar}$ respectively, (c) the positivesequence terminal current, and (d) overcurrent protection tripping signal.

Fig. 2 shows the response of the three-phase motor load on bus 860 to a $200 \mathrm{~ms}$ three-phase fault at its terminals; the results shown here were obtained using EMTP. As shown, following the fault at $\mathrm{t}=5 \mathrm{~s}$, the terminal voltage shown in Fig. 2(a) drops and the motor stalls. Consequently, the real and reactive power consumption and the positive-sequence terminal current of the motor increase as shown in Fig. 2(b), (c). Because the current exceeds $5 \mathrm{pu}$ for more than $1 \mathrm{~s}$ during the stalling mode, the overcurrent protection issues a tripping signal to disconnect the motor as shown in Fig. 2(d). The motor gets reconnected after 25 s (not shown.)

\section{B. Modified Model of the Electric Power System}

The model of the Electric Power System (EPS) has also been modified using the parameters of Table 1. The changes made to the EPS model alter the previously obtained power flow solution, as presented in Table 2 .

\section{Modified Control Schemes}

In the previous version of the ADN model [7], the models of the EPS and DER units of the EMTP-RV and SSN implementations only match in terms of their output in steadystate real and reactive powers. The latest version presented in this paper improves the matching between these models to achieve a more consistent transient response. To that end, the control schemes of the EPS and wind generator models of the EMTP-RV implementation have been modified to match those of the SSN version. The EPS model uses the parameters of Table 1 .

TABLE 1. PARAMETERS OF THE NEW EPS MODEL.

\begin{tabular}{|l|l|l|}
\hline Component & Parameter & Value \\
\hline \multirow{5}{*}{} & Nominal power & $1000 \mathrm{MW}$ \\
\cline { 2 - 3 } & Nominal voltage & $231 \mathrm{kV}$ RMSLL \\
\cline { 2 - 3 } & Xd & $1.8 \mathrm{pu}$ \\
\cline { 2 - 3 } & Xd' & $0.3 \mathrm{pu}$ \\
\cline { 2 - 3 } & Xd" & $0.25 \mathrm{pu}$ \\
\cline { 2 - 3 } & Xq & $0.55 \mathrm{pu}$ \\
\cline { 2 - 3 } & Xq' & $0.25 \mathrm{pu}$ \\
\cline { 2 - 3 } & Xq" & $0.2 \mathrm{pu}$ \\
\cline { 2 - 3 } & Tdo' & 8 \\
\cline { 2 - 3 } & Tdo" & 0.03 \\
\cline { 2 - 3 } & Tqo' & 0.4 \\
\cline { 2 - 3 } & Tqo" & 0.05 \\
\cline { 2 - 3 } & Stator resistance & $0.0025 \mathrm{pu}$ \\
\cline { 2 - 3 } & Inertia coefficient & $6.5 \mathrm{~s}$ \\
\cline { 2 - 3 } & Friction factor & $0 \mathrm{pu}$ \\
\cline { 2 - 3 } & Pole pairs & 4 \\
\cline { 2 - 3 } & Rotor type & Round \\
\hline Exciter & Type & DC1A \\
\hline Governor-Turbine & Type & IEEEG1 \\
\hline PSS & Type & PSS1A \\
\hline
\end{tabular}

(a)

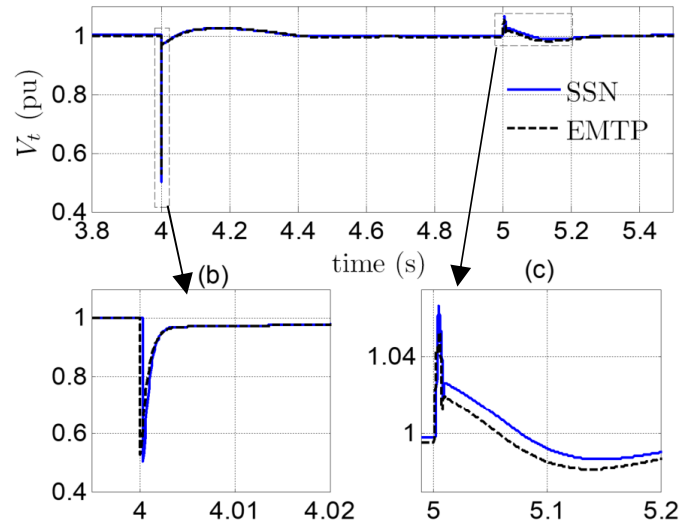

(d)

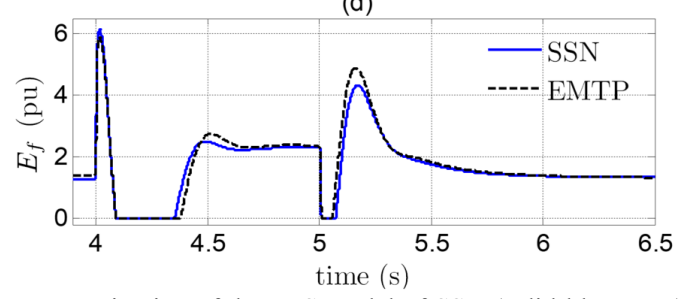

Fig. 3. Cross-examination of the EPS model of SSN (solid-blue trace) against EMTP-RV (dashed-black trace) under a load change at $\mathrm{t}=4 \mathrm{~s}$ and $\mathrm{t}=5 \mathrm{~s}$ : (a) positive-sequence terminal voltage in pu, (b) zoomed view of the positivesequence terminal voltage during the first load change at $\mathrm{t}=4 \mathrm{~s}$, (c) zoomed view of the positive-sequence terminal voltage during the second load change at $\mathrm{t}=5 \mathrm{~s}$, and (d) electric field in pu.

Fig. 3 cross-examines the response of the EPS model from SSN (solid-blue trace) against EMTP-RV (dashed-black trace) under two load changes at $\mathrm{t}=4 \mathrm{~s}$ and $\mathrm{t}=5 \mathrm{~s}$. The peak value of the waveform of Fig. 3(b) at $4 \mathrm{~s}$ is different by $4.7 \%$ in the SSN and EMTP implementations. This difference is $1.5 \%$ in Fig. 3(c) for the peak value at $5 \mathrm{~s}$ and $11.5 \%$ in Fig. 3(d) for the peak value at $5.2 \mathrm{~s}$. The results demonstrate an acceptable agreement between the two models.

Fig. 4 cross-examines the response of the wind generator 
model of EMTP-RV versus SSN under a load change at $\mathrm{t}=5 \mathrm{~s}$ showing the $\mathrm{d}$ - and q-axis components of terminal current in pu. For the first peak at $5.002 \mathrm{~s}$ the difference between the results of EMTP and SSN is $12.5 \%$ while this difference is $14.5 \%$ for the second peak at $5.005 \mathrm{~s}$. It should be mentioned that the results of Fig. 4 show some inconsistencies due to modeling differences including different PLL models and signal filtering schemes.
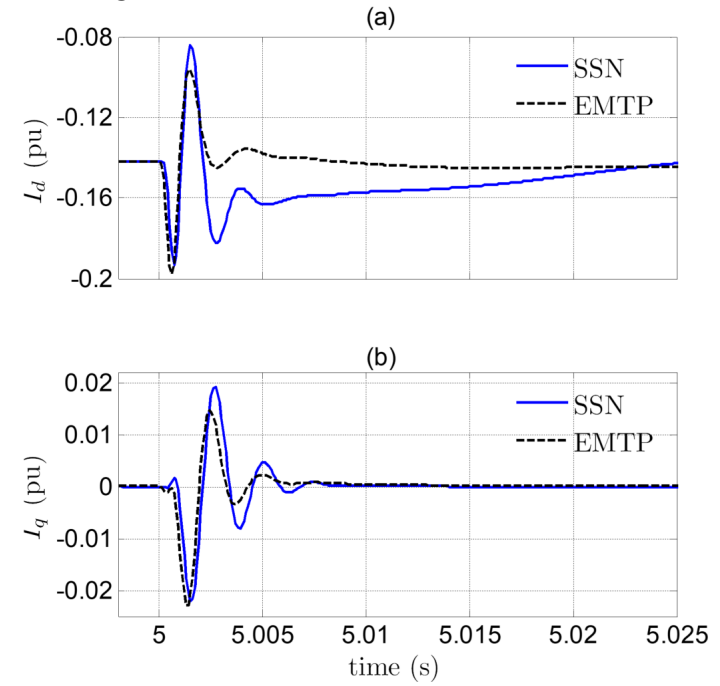

Fig. 4. Cross-examination of the model of the wind generator of SSN (solidblue trace) against EMTP-RV (dashed-black trace) under a load change at $\mathrm{t}=5 \mathrm{~s}$ : (a) d-axis terminal current in pu and (b) q-axis terminal current in pu.

\section{SimULATION RESULTS}

Table 2 presents the power flow solution of the latest version of the ADN obtained in EMTP. This solution slightly differs from that of the previous version [7] due to the change in the EPS model. As shown, the voltage amplitude is between $0.99 \mathrm{pu}$ and $1.06 \mathrm{pu}$ indicating an acceptable solution. The voltage amplitudes are generally larger than those of the previous version [7] due to the removal of the transformer interfacing the synchronous generator of the EPS to the rest of the grid. The power flow solution illustrates that the ADN is unbalanced due to the presence of unbalanced three-phase loads, single-phase loads, and single-phase residential PV units.

To examine time-domain results, the fault scenario of [7] has been repeated here which studies a 6-cycle three-phase bolted fault on the MV feeder at bus 858 at $\mathrm{t}=50 \mathrm{~s}$ followed by the opening of the three-phase MV recloser at the feeder section 832-858 after 2 cycles. The opening and re-closure times of the MV breaker are 50.033s and 50.2331s, respectively. Fig. 5 shows the rms value of the voltage of phase of bus 832 in response to the fault obtained from the SSN (solid-blue trace) and the EMTP (dashed-black trace) models. As shown, the voltage waveforms match more closely compared to the previous version (see Fig. 3 (a) of [7]) specifically with regards to the voltage undershoot due to the re-closure of the breaker at $\mathrm{t}=50.2331 \mathrm{~s}$. The peak value of the waveforms shows a difference of $1.0 \%$ in the overshoot at $50.05 \mathrm{~s}$ and $1.2 \%$ in the undershoot at $50.3 \mathrm{~s}$, respectively

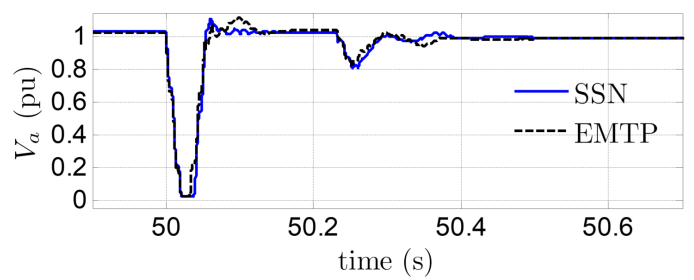

Fig. 5. Voltage (phase ' $a$ ') of bus 832 of the MV section in response to a three-phase bolted fault on bus 858 at $\mathrm{t}=50 \mathrm{~s}$ obtained from the SSN (solidblue trace) and the EMTP-RV (dashed-black trace) models.

To further improve the consistency of results, the following changes need to be made in future implementations:

- Matching PV models: in the latest version, the PV units of the EMTP-RV and SSN implementations provide the same steady-state output powers, nonetheless, their transient response differs significantly. This is due to the difference in the control scheme parameters and power circuit of the PV unit models. Future implementations of the ADN may improve the consistency of results by matching the model of the PV units.

- Load models: The SSN implementation models the loads as constant-power, constant-current, or constant-impedance loads whereas the latest EMTP implementation models the loads as constant-impedance for simplicity. These load models are available in EMTP and can be incorporated in future implementations of the ADN to match the results more closely.

\section{CONCLUSIONS}

Expanding upon the authors' previous work [5]-[8], this paper has presented the latest version of an Active Distribution Network test system for Smart Grid, control, protection, and dynamic studies. The latest version includes additional protection functions and modifications to reduce modeling differences of the EMTP and SSN implementations. The paper has qualitatively shown that these modifications have resulted in a closer match between the results of the two implementations compared to the old version. The presented simulation results serve as a further step towards software-tosoftware validation of the test $\mathrm{ADN}$.

\section{APPENDIX}

Table 2 presents the power-flow solution of the ADN obtained in EMTP.

\section{REFERENCES}

[1] CIGRE Task Force C6.04.02, CIGRE Tech. Brochure, "Benchmark systems for network integration of renewable and distributed energy resources," 2011.

[2] W.H. Kersting, "Radial distribution test feeders," in IEEE PES Winter Meeting, 2001.

[3] http://www.ewh.ieee.org/soc/pes/dsacom/testfeeders/index.html

[4] J.S. Savier, D. Das, "Impact of network reconfiguration on loss allocation of radial distribution system," IEEE Trans. Power Del., vol. 22, no. 4, pp. 2473-2480, Oct. 2007.

[5] H. Hooshyar, F. Mahmood, L. Vanfretti, and M. Baudette, "Specification, implementation, and hardware-in-the-loop real-time simulation of an active distribution grid," Sustainable Energy, Grids and Networks, vol. 3, pp.36-51 Sep. 2015.

[6] H. Hooshyar, L. Vanfretti, C. Dufour, "Delay-free parallelization for real-time simulation of a large active distribution grid model," in Proc. IEEE IECON, Italy, Oct. 2016. 
[7] A. Haddadi, H. Hooshyar, J. Mahseredjian, C. Dufour, and L. Vanfretti, "A first step towards the implementation and software-to-software validation of an active distribution network model," in International Conference on Power Systems Transients (IPST), Seoul, South Korea, 2017.

[8] Hossein Hooshyar and Luigi Vanfretti (2016). FP7-IDE4LKTHSmarTSLab-ADN-RTModel: First Release [Data set]. Sustainable Energy, Grids and Networks. Zenodo. http://doi.org/10.5281/zenodo.61183
Available at Github: https://github.com/SmarTS-Lab/FP7-IDE4LKTHSmarTSLab-ADN-RTModel

[9] C. Dufour, J. Mahseredjian and J. Bélanger: "A combined state-space nodal method for the simulation of power system transients," IEEE Trans. Power Del., vol. 26, no. 2, pp. 928-935, Apr. 2011.

[10] https://www.mathworks.com/help/physmod/sps/

TABLE 2

THREE-PHASE POWER FLOW SOLUTION OF THE ADN IN EMTP-RV

\begin{tabular}{|c|c|c|c|c|c|c|c|c|c|c|c|c|c|}
\hline \multirow[b]{2}{*}{ Bus } & \multicolumn{2}{|c|}{ Phase a } & \multicolumn{2}{|c|}{ Phase b } & \multicolumn{2}{|c|}{ Phase c } & \multirow[b]{2}{*}{ Bus } & \multicolumn{2}{|c|}{ Phase a } & \multicolumn{2}{|c|}{ Phase b } & \multicolumn{2}{|c|}{ Phase c } \\
\hline & $\begin{array}{c}\mathrm{V} \\
(\mathrm{pu})\end{array}$ & $\begin{array}{l}\text { Phase } \\
\text { angle } \\
\text { (deg) }\end{array}$ & $\begin{array}{c}\mathrm{V} \\
(\mathrm{pu})\end{array}$ & $\begin{array}{l}\text { Phase } \\
\text { angle } \\
\text { (deg) }\end{array}$ & $\begin{array}{c}\mathrm{V} \\
(\mathrm{pu})\end{array}$ & $\begin{array}{l}\text { Phase } \\
\text { angle } \\
\text { (deg) }\end{array}$ & & $\begin{array}{c}\mathrm{V} \\
(\mathrm{pu})\end{array}$ & $\begin{array}{l}\text { Phase } \\
\text { angle } \\
\text { (deg) }\end{array}$ & $\begin{array}{c}\mathrm{V} \\
(\mathrm{pu})\end{array}$ & $\begin{array}{l}\text { Phase } \\
\text { angle } \\
\text { (deg) }\end{array}$ & $\begin{array}{c}\mathrm{V} \\
(\mathrm{pu})\end{array}$ & $\begin{array}{l}\text { Phase } \\
\text { angle } \\
\text { (deg) }\end{array}$ \\
\hline 100 & 1.05 & 0 & 1.05 & -120 & 1.05 & 120 & 741 & 1.03 & 18.4 & 1.03 & -101.6 & 1.03 & 138.3 \\
\hline 101 & 1.02 & -8.1 & 1.02 & -128.1 & 1.02 & 111.9 & 742 & 1.04 & 18.3 & 1.03 & -101.7 & 1.03 & 138.4 \\
\hline 102 & 1.02 & -9.4 & 1.02 & -129.4 & 1.02 & 110.6 & 744 & 1.04 & 18.3 & 1.04 & -101.7 & 1.04 & 138.4 \\
\hline 104 & 1.02 & -9.6 & 1.02 & -129.6 & 1.02 & 110.5 & 800 & 1.02 & 19.4 & 1.02 & -100.5 & 1.02 & 139.7 \\
\hline 105 & 1.03 & -8.1 & 1.03 & -128.1 & 1.03 & 111.9 & 802 & 1.02 & 19.4 & 1.02 & -100.5 & 1.02 & 139.7 \\
\hline 106 & 1.02 & 19.4 & 1.02 & -100.5 & 1.02 & 139.7 & 806 & 1.02 & 19.3 & 1.02 & -100.5 & 1.02 & 139.7 \\
\hline 701 & 1.04 & 18.3 & 1.04 & -101.6 & 1.04 & 138.3 & 808 & 1.01 & 19.1 & 1.01 & -100.7 & 1.02 & 139.5 \\
\hline 702 & 1.04 & 18.3 & 1.04 & -101.7 & 1.03 & 138.3 & 810 & n.a. & n.a. & 1.01 & -100.7 & n.a. & n.a. \\
\hline 707 & 1.03 & 18.3 & 1.03 & -101.7 & 1.03 & 138.4 & 820 & 1.03 & 18.6 & n.a. & n.a. & n.a. & n.a. \\
\hline 708 & 1.03 & 18.4 & 1.03 & -101.6 & 1.03 & 138.4 & 822 & 1.03 & 18.6 & n.a. & n.a. & n.a. & n.a. \\
\hline 709 & 1.03 & 18.4 & 1.03 & -101.6 & 1.03 & 138.4 & 824 & 1.03 & 18.6 & 1.04 & -101.3 & 1.04 & 139.0 \\
\hline 710 & 1.03 & 18.4 & 1.03 & -101.6 & 1.03 & 138.4 & 826 & n.a. & n.a. & 1.04 & -101.3 & n.a. & n.a. \\
\hline 711 & 1.03 & 18.4 & 1.03 & -101.6 & 1.03 & 138.4 & 828 & 1.03 & 18.6 & 1.04 & -101.3 & 1.04 & 139.0 \\
\hline 712 & 1.04 & 18.3 & 1.04 & -101.7 & 1.03 & 138.4 & 830 & 1.03 & 18.5 & 1.04 & -101.4 & 1.04 & 138.8 \\
\hline 713 & 1.04 & 18.3 & 1.04 & -101.7 & 1.03 & 138.3 & 832 & 1.05 & 18.3 & 1.06 & -101.6 & 1.06 & 138.4 \\
\hline 714 & 1.03 & 18.3 & 1.03 & -101.7 & 1.03 & 138.3 & 834 & 1.05 & 18.3 & 1.06 & -101.7 & 1.06 & 138.3 \\
\hline 718 & 1.03 & 18.3 & 1.03 & -101.7 & 1.03 & 138.4 & 836 & 1.05 & 18.3 & 1.06 & -101.7 & 1.06 & 138.3 \\
\hline 730 & 1.04 & 18.4 & 1.03 & -101.6 & 1.03 & 138.4 & 852 & 1.01 & 18.3 & 1.02 & -101.6 & 1.03 & 138.4 \\
\hline 732 & 1.03 & 18.4 & 1.03 & -101.6 & 1.03 & 138.4 & 854 & 1.03 & 18.5 & 1.04 & -101.4 & 1.04 & 138.8 \\
\hline 733 & 1.03 & 18.4 & 1.03 & -101.6 & 1.03 & 138.4 & 856 & n.a. & n.a. & 1.04 & -101.4 & n.a. & n.a. \\
\hline 734 & 1.03 & 18.4 & 1.03 & -101.6 & 1.03 & 138.4 & 858 & 1.05 & 18.3 & 1.06 & -101.6 & 1.06 & 138.3 \\
\hline 735 & 1.03 & 18.4 & 1.03 & -101.6 & 1.03 & 138.4 & 860 & 1.05 & 18.3 & 1.06 & -101.7 & 1.06 & 138.3 \\
\hline 736 & 1.03 & 18.4 & 1.03 & -101.6 & 1.03 & 138.3 & 862 & 1.05 & 18.3 & 1.06 & -101.7 & 1.06 & 138.3 \\
\hline 737 & 1.03 & 18.4 & 1.03 & -101.7 & 1.03 & 138.4 & 864 & 1.05 & 18.3 & n.a. & n.a. & n.a. & n.a. \\
\hline 738 & 1.03 & 18.4 & 1.03 & -101.7 & 1.03 & 138.4 & 888 & 1.04 & 18.1 & 1.05 & -101.8 & 1.05 & 138.2 \\
\hline 740 & 1.03 & 18.4 & 1.03 & -101.6 & 1.03 & 138.3 & $890 / 799$ & 1.00 & 18.3 & 1.00 & -101.6 & 1.01 & 138.4 \\
\hline
\end{tabular}

